\title{
Experiencia con un pueblo indígena de reciente contacto en el Valle de Yavarí1
}

\author{
Experience with a recent contacted indígenous people \\ in the Yavari Valley
}

Sanderson Castro Soares de Oliveira

\begin{abstract}
Resumo:
En este articulo presento una discusión sobre el contacto de pueblos indígenas y la situación de reciente contacto. Destaco cuestiones centrales para el entendimiento de la definición de "Indigenismo" en Brasil, basado en Ramos (1998:7) y focalizando el contacto y el postcontacto de los índigenas Korúbo. Evidencio tambié el rol del Estado, la subjetividad de los actores y la naturaleza incompleta de las acciones y propuestas de políticas públicas.
\end{abstract}

Palavras-chave: Pueblos de reciente contato, "Indigenismo", Contacto y post-contacto, Pueblo Korúbo, Políticas públicas

\begin{abstract}
:
I present a discussion on the contact of indigenous people and the situation of recent contact. Based on Ramos (1998:7), I highlight central issues for the understanding of what is defined as "Indigenismo" in Brazil, focusing on the case of the contact and post-contact of the Korubo Indians. I also show the role of the State, the subjectivity of the actors, the incomplete nature of the actions and proposals of public policies.
\end{abstract}

Keywords: Recent contacted people, "Indigenismo", Contact and post-contact, the Korúbo people, Public policies

\section{El concepto de contacto}

Al hablar de experiencias de contacto, debemos aclarar que estas se constituyen a partir de un sentido histórico y de un contexto social determinado en el tiempo. Las experiencias de contacto en Brasil varían mucho en sentido y en práctica, como lo apunta Freire (2005) al analizar las estrategias de contacto a partir de la historia de los principales agentes del contacto en el siglo XX.

Ramos (1999:2) subraya el efecto negativo del contacto al comentar la discusión antropológica sobre el tema. Para esta autora, no importa el nombre

\footnotetext{
1 Versión revisada de la comunicación presentada en el Entrenamiento Regional en aspectos técnicos para la ubicación, delimitación y protección de los Pueblos Indígenas en Aislamiento Voluntario y la Formulación de planes de contigencia para poblaciones en Contacto Inicial (PIAVCI).
} 
que utilicemos para la discusión, pero es necesario enfocarse en el rol del Estado en esta acción. En sus palabras:

não é possível deixar de lado o papel do Estado nacional quando se quer compreender o impacto secular daquilo que Paul Ricoeur chamou de "evento fundador" (Ricoeur 1978) ou do que Veena Das caracteriza como "evento crítico" (Das 1995), ou seja, a presença ubíqua e devastadora dos brancos na vida dos povos indígenas.

Al mismo tiempo, en otro artículo la misma autora resalta la subjetividad de los "actores sociales" y su influencia en la creación de políticas públicas que afectan a los indígenas.

Trata-se de como o peso de certas personalidades do indigenismo brasileiro, pela força do carisma de suas personas públicas, deixa-os à vontade para conceber ações de circunstância, muitas vezes motivadas por reações emocionais, e depois transformar essas ações e reações em praxis oficial. (Ramos 1998:7)

Estos me parecen elementos que debemos tener en cuenta antes de empezar la discusión acerca del contacto y el contacto inicial, pues se trata de cuestiones centrales para entender lo que definimos en Brasil como "Indigenismo" y que Ramos (1998:7) define en su sentido más amplio "como um complexo edifício ideológico construído sobre diferenças étnicas, uma obra que nunca se completa." Es necesario subrayar la visión inacabada del indigenismo, pues si esta es una obra que nunca se completa debemos conformarnos con la imposibilidad de actuar dentro del marco de un indigenismo con respuestas definitivas.

Los elementos subrayados arriba, a partir de las discusiones de Alcida Rita Ramos, parecen estar presentes también en la historia del contacto y del postcontacto de los Korúbo Recién Contactados. La discusión aquí presentada intenta contextualizar el contacto y el post-contacto Korúbo históricamente, así como evidenciar el rol del Estado, la subjetividad de los actores y la naturaleza incompleta de las acciones y propuestas de políticas públicas.

En las dos primeras secciones se presenta el contexto histórico del contacto y del período posterior caracterizado como post-contacto. Se busca en estas secciones dar a conocer al público quiénes son los Korúbo y cómo se relacionan con la política pública para indígenas aislados y en contacto inicial.

\section{El contacto Korúbo:}

El grupo Korúbo recién contactado está compuesto por 31 personas $^{2}$ que se

\footnotetext{
${ }^{2}$ Estos datos dicen respecto al año de 2012 y se refieren al grupo de Mayá. Después de la ponencia hubo 2 contactos que resultaron en un crecimiento sustancial en la comunidad
} 
encuentran en contacto permanente con la sociedad nacional brasileña desde 1996. Sin embargo, reconocer esta fecha no significa que este grupo estuvo aislado durante toda su historia. En efecto, no es difícil acceder a evidencias de contactos de grupos Korúbo con la sociedad nacional y con otros pueblos (cf. Coutinho Jr.1998; Arisi 2005; Oliveira 2009).

En el caso Korúbo los contactos fueron marcados por una gran violencia, al punto de ser caracterizados por Coutinho Jr. (Op. Cit.: 54) como un "martirologio". Dicho autor relata que los Korúbo se comportaban como "una sociedad en guerra" (id. Ibídem), comportamiento adoptado por los Korúbo frente a las amenazas que sufrían - por parte de madereros, pescadores y otros no indígenas que habitan hoy las cercanías de la Tierra Indígena - y que ayudó a crear y consolidar su fama de pueblo violento, guerrero y, por lo tanto, amenazador. Esta fama persiste hasta el día de hoy e interfiere en la implementación de políticas con respecto a este pueblo, ya sea en su relación con grupos aislados, con otros grupos contactados o con los representantes del Estado.

El contacto oficial del grupo Korúbo - hito para una política de contacto reciente para ellos - ocurrió después del inicio de la política de no contacto. (cf. Oliveira 2009; Arisi 2005). Si observamos detenidamente el caso Korúbo, podemos observar una fuerte diferencia entre la política instituida por el Estado y la práctica en el campo (cf. Arisi 2005; Coutinho Jr. 1998; Oliveira 2009), pero en líneas generales aún podemos observar que las políticas garantizaron:

a) que ya no se aplicaba una visión integracionista del contacto,

b) que el contacto solo se realizó por el peligro inminente de extinción de todo el grupo.

c) que ahora los Korúbo dejaban de pertenecer a una categoría llamada aislados y que todo lo siguiente se daba dentro de una nueva categoría.

La cuestión de la diferencia entre la praxis indigenista y las normas del Estado tiene implicaciones para el trabajo con los Korúbo. Pero antes de tratar este tema tenemos que observar que estamos hablando de categorías ajenas a los indígenas o que ellos interpretan desde otro punto de vista. Si desde afuera se aduce que los Korúbo fueron contactados para garantizar la supervivencia del grupo, sus relatos no soportan dicha afirmación. Es común escuchar en sus recuentos sobre el contacto que, desde su punto de vista, ellos iban a acabar con los no-indígenas y no lo contrario. Además, siempre subrayan que su

Korubo que llega hoy a más de 90 personas. Aún que el grupo de Mayá empezó a relacionarse y añadió personas por matrimonio a sus relaciones, el número del grupo, sin considerar los nuevos contactos no está muy distinto actualmente. 
supervivencia no estaba amenazada.

Es común también que los Korúbo relaten que fueron ellos quienes se acercaron a los no-indígenas por su deseo de estar junto a nosotros. Esta afirmación resalta el protagonismo del grupo en su historia y su rol como agente desencadenador de acciones. En el caso del contacto este rol activo es también registrado en varios otros grupos (cf. Albert y Ramos 2002; Carneiro da Cunha 1992; Arisi 2005).

Además, las categorías "contactado", "en contacto inicial” y "aislados" son desconocidas para el indígena, que de un día a otro - o dentro de un par de días o meses - pasa a ser clasificado dentro de uno de estos grupos y tiene que convivir con estas nuevas categorías. ¿Cómo decirle a un grupo que ellos están sujetos a una política diferenciada por parte del gobierno porque están clasificados dentro de una de estas categorías?

En términos de traducción los Korúbo hacen una diferenciación entre indio txuka ${ }^{3}$ (indio nuevo) e indio xëni ('índio viejo'). Estas categorías reflejan el marco del contacto y el alejamiento temporal frente a ello como un indicador de diferencias entre las situaciones de los distintos grupos indígenas. Esta percepción aparece relacionada a muchas cuestiones que nos hacen pensar que los indígenas están conscientes de las diferentes políticas públicas desarrolladas según las categorías elaboradas por el gobierno. Por otro lado, esto no presupone una equivalencia terminológica entre las palabras en portugués y las traducciones en Korúbo.

Otra cosa que nos llama la atención es que los Korúbo utilizan el término "indios," una categoría externa que los incluye en un grupo abstracto que tampoco es propio, pero que es altamente operativo y justifica incluso la propia acción indigenista. Es decir, los Korúbo empiezan a incluirse dentro de la categoría de indígenas aunque no se observe una categoría semejante en el uso actual de su lengua. Además, los contextos en que se inserta el uso de los términos citados sugiere que los Korúbo quieren nivelarse con otros grupos que conocen y con quienes se relacionan. Es común, por ejemplo, que ellos cuestionen su estatus de "indio txuka" cuando quieren tener acceso a materiales, instrumentos o beneficios no materiales que ya tienen los "indio xëni"4.

Esta tensión entre indígenas nuevos y viejos es también ambivalente, pues sirve para garantizar acciones diferenciadas que los indígenas Korúbo perciben como ventajas. Por ejemplo, es común que aboguen por el estatus de "indios nuevos" cuando quieren que la FUNAI los acompañe en acciones de

\footnotetext{
${ }^{3}$ Utilizan también la palavra paxa, que me parece un préstamo del Matís.

${ }^{4}$ Esta situación de acceso a los materiales, instrumentos y recursos occidentales se está cambiando rápidamente desde 2007, cuando empecé a trabajar con ellos. De especial atención, se nota la adquisición y dominio de los motores para barcos, popularmente conocidos como peque-peque en la región.
} 
negociación de su producción en la ciudad. Es interesante notar que los Korúbo suelen utilizar en estas discusiones un discurso que podemos resumir, en líneas generales, como de vulnerabilidad frente a nuestros códices y, principalmente, frente a nuestro sistema económico y político.

La discusión sobre las percepciones de los Korúbo frente al proceso de contacto y post-contacto no se agota en la simple discusión terminológica, como se ha hecho aquí, sino que nos interesa la discusión sobre qué es un pueblo indígena en contacto inicial. Frente a la multiplicidad de definiciones por parte de los diferentes agentes gubernamentales en los distintos países y la dificultad de utilizar directamente las categorías indígenas, presento acá la definición expuesta en el documento final de las consultas realizadas por OACNUDH en Bolivia, Brasil, Colombia, Ecuador, Paraguay, Perú y Venezuela (OACNUDH 2012), que en su título revela su objetivo de orientar las Directrices de Protección para los Pueblos Indígenas en Aislamiento y en Contacto Inicial de la Región Amazónica, el Gran Chaco y la Región Oriental de Paraguay.

Los pueblos en contacto inicial son pueblos que mantienen un contacto reciente con la población mayoritaria; pueden ser también pueblos que a pesar de mantener contacto desde tiempo atrás, nunca han llegado a conocer con exactitud los patrones y códigos de relación de la población mayoritaria. Esto puede deberse a que estos pueblos mantienen una situación de semi aislamiento, o a que las relaciones con la población mayoritaria no son permanentes, sino intermitentes. Los pueblos "en contacto inicial" son pueblos que previamente permanecían "en aislamiento" y que bien forzados por agentes externos, bien por decisión del grupo, o por factores de otro tipo entran en contacto con la población mayoritaria. . De esta manera podríamos hablar bajo la categorización de pueblos en contacto inicial de pueblos en contacto intermitente, en contacto permanente, en peligro de extinción y pueblos extinguidos. Dado que no existe un consenso a nivel internacional sobre cuestiones tales como cuáles son los criterios para dar por terminada una situación de aislamiento y cuándo comienza la de contacto inicial o cuándo se da por terminada la situación de contacto inicial, es necesario dar mayor peso a criterios adicionales relacionados con la situación de alta vulnerabilidad (enfermedades, reducción territorial, etc.), en que se encuentran los indígenas en mención. Esa situación puede persistir aún después de muchos años de contactos sostenidos con miembros de la sociedad mayoritaria y mientras persista el riesgo de extinción debido a los problemas generados por dicha sociedad y las consecuencias generadas desde el momento del contacto.

Esta definición no es exactamente la misma utilizada en Brasil y tampoco 
coincide con lo que piensan los Korúbo, consideradas las distancias culturales entre lo que piensan ellos y una política pública general. Pero podemos encontrar concordancias entre las visiones aquí mencionadas. El contacto aparece en todas ellas como un determinante en las varias categorizaciones presentadas. El tiempo, aunque no sea determinante, también aparece en las definiciones. Y nos falta resaltar la vulnerabilidad, que no aparece directamente en los discursos Korúbo, pero que pueden ser fácilmente percibidas a partir de discursos como los citados antes sobre su falta de dominio de nuestros códigos, o también en cuestiones de salud, como cuando dicen "si me vuelvo al monte, me muero por cuestiones de salud" o "antiguamente no teníamos las enfermedades de los blancos, pero ahora ya no podemos, pues, si nos vamos, nos morimos."

\section{Volviéndose recién contactados:}

Después del contacto en 1996 la asistencia a los Korúbo sigue dentro del Departamento de Índios Isolados de $\mathrm{FUNAI}^{5}$, lo que significa una atención diferenciada. Sin embargo, el Estado todavía no tenía instrumentos legales y formales diferenciados de actuación frente a los grupos en contacto inicial. Esta política diferenciada de actuación frente a los Korúbo resulta en la creación del Frente de Contacto Valle de Yavarí como institución ejecutora de las acciones gubernamentales.

En el año 2000, una nueva orientación política frente a la situación de contacto y a los indígenas aislados cambia los nombres de la dirección y del frente ejecutor, que pasan a llamarse:

- Coordenação Geral de Índios Isolados - CGII

- Frente de Proteção Etno-Ambiental Vale do Javarí.

Infelizmente, aunque esta nueva orientación muestre más coherencia con la política de no-contacto al omitir el término "contacto" en su título y enfatizar la protección, el órgano indigenista de Brasil sigue sin destacar los indígenas de reciente contacto en su organograma y en su legislación y no se crea una coordinación específica para atención a esos grupos.

En la práctica la DII y su sucesora, la CGII, siguen acompañando a cuatro grupos en situaciones especiales que llaman en su práctica "de reciente contacto," "recién contactados" o con términos semejantes. Esta experiencia con los grupos Kanoê y Akuntsú (Rondônia), Suruahá (Amazonas), Guajá (Maranhão), Zo'é (Pará) y con los Korúbo (Amazonas) empieza lo que podemos llamar el embrión de una política para indígenas de reciente contacto.

En los diferentes casos, todos cuentan con grupos de asesores en temas de lingüística y antropología, y algunos en temas de salud. Hay que resaltar,

${ }^{5}$ Este departamento fué creado cuando del comienzo de la política de no contacto, en 1987. 
entretanto, que el ingreso y la actuación de estas personas en los diferentes procesos en curso se da de manera distinta, observándose las particularidades de cada caso.

En el caso Korúbo, el coordinador del Frente de Protección que ingresa en 2005, Antenor Vaz, es el responsable de la decisión del ingreso de un lingüista y un antropólogo como asesores para este grupo. Es solo en 2007, a partir de la decisión de esta meta que yo paso a ser uno de los actores sociales que se relacionan con el grupo Korúbo de reciente contacto.

Paralelo a esto, se empiezan a delinear los lineamientos generales de la política para indígenas en contacto inicial que serán los determinantes de una política pública que aún no se encuentra claramente definida en Brasil. Este proceso crea algunos reflejos en la práctica y en el organograma del órgano indigenista brasileño. En 2009, la política para indígenas de reciente contacto pasa a ser, por primera vez, reconocida dentro de la reestructuración de FUNAI. La creación de una coordinación específica se dio por una ley y agregó al nombre de la antigua coordinación el nombre "Recente Contato", pasando a llamarse ahora "Coordenação Geral de Índios Isolados y Contacto Inicial" - CGIIRC.

En la práctica, la orientación de la Coordinación para el trabajo con grupos en contacto inicial se da por una serie de capacitaciones y formulaciones internas que orientan el tema, pero que se diferencian según los ejecutores y las interpretaciones locales. Una importante publicación que sintetiza las orientaciones sobre el tema sale por primera vez en 2011, bajo el título "Politica de Estado: de La Tutela a la Política de Derechos - ¿Una Cuestion Resuelta?" (Vaz 2011).

Entre otras cuestiones que nos parecen interesantes el documento resalta el rol del Estado como protector de los derechos indígenas y determina los principios básicos que deben orientar la actuación con los indígenas aislados y en contacto inicial, como sigue abajo:

1. Principio de autodeterminación:

2. Principio de libre acceso, movilidad y usufructo de su territorio:

3. Principio de salud plena:

4. Principio de comunicación plena:

5. Principio de alteridad:

6. Principio de precaución:

En 2011 se dan también los primeros intentos por delinear el Programa Korúbo, que servirá de marco orientador de las acciones prácticas con este pueblo 
y que tiene como base los principios expuestos arriba. Además, el programa tiene como premisa los "procesos educativos", que deben estar presentes en todas las acciones realizadas y en todas sus líneas de actuación.

Otra vez volvemos a hablar de políticas públicas y nos olvidamos de los Korúbo. ¿Que pasó con los Korúbo con todas estas acciones por parte de los actores sociales? Bueno, en cuanto al cambio de nombres de DII a CGII y CGIIRC y de FCVJ a FPEVJ, creo que no pasó mucho, pues aún hoy entre los otros indígenas y la población ribereña la FPEVJ sigue siendo la FCVJ, o Frente de Contacto. Los Korubo, por su parte, generalmente se refieren simplemente al Frente o Base, sin importarles mucho si es de contacto o de protección, aunque ya se les haya explicado varias veces el cambio de enfoque.

Desde el punto de vista de las acciones, resalto la introducción de nuevos elementos y la dependencia cada vez más acentuada de una medicina ajena frente a nuevos agravios a su salud como la gripe y la malaria. Como se ha explicitado antes, estas acciones parecen ir en contra de la política delineada y defendida por el gobierno, pero aparecen dos elementos importantes que caracterizan la "presencia ubicua" del blanco en la vida indígena, resultante del contacto, como lo subrayado por Ramos (Op. Cit.) más arriba.

¿Y qué puedo decir sobre el período de 2007 al 2011 en que acompañé más detenidamente al grupo Korúbo? Sobre esto pretendo ocuparme en la próxima sección y presentar algunas reflexiones principalmente sobre cómo las cuestiones prácticas se relacionan con los principios delineados para orientarlas.

\section{Nukun latkute txoak: ¿Llegó nuestro blanco!}

Mi llegada donde los Korúbo se dio tras una serie de negociaciones y demandas de ellos, pero también por intereses del órgano de gobierno en tener una relación más directa con los miembros del grupo. Como me explicaron los actores sociales que me llevaron allá, yo debía ir para aprender la lengua de los Korúbo y también para enseñarles un poco de portugués, que era su principal demanda.

Diferentemente de lo que uno puede esperar, Xikxu Korúbo me relata que él le pidió a Antenor que le enviara un Latkute (o nawa) para enseñarles a sus hijos la lengua portuguesa y otras cosas. Es impresionante su narrativa, pues dentro de ella ya tenía incluso planeado un espacio donde yo viviría en la aldea y una casa que iba a empezar a construir.

Otra vez se nota el protagonismo indígena en la construcción de la historia, de la misma forma que pude observar en todo lo que pasó en los últimos 5 años. No hay un caso en que yo pueda decir que no tuve que negociar con el grupo. Pero aceptar que el grupo tiene derechos no quiere decir que en la práctica se acepten todas sus demandas. El espacio de negociación entre lo que quieren ellos, lo que quiere el gobierno y lo que es posible es exactamente el espacio en 
el que se llevan a cabo las acciones de los actores sociales en el campo.

Decidir, por ejemplo, entre el principio que les garantiza a los Korúbo el usufructo de su tierra y el principio de precaución es exactamente lo que está en juego cuando uno piensa si debe aceptar la invitación de tener una casa en la aldea o no. En este caso, opté por no tenerla y, de verdad, Xikxu nunca me la construyó, pero tampoco lo incentivé o le di señales de que viviría dentro de la aldea.

Además, hoy día soy capaz de percibir que llevar a cabo el principio de la comunicación plena es de alguna forma ir en contra del principio de alteridad, pues para que uno aprenda bien la lengua del otro hay que convivir, y muy de cerca, y no es posible convivir sin alterar nada. Pero, por otro lado, como muy bien nos esclarece Laraia (1986) la cultura no es un concepto fijo, es dinámica y cambia con el tiempo y con el contacto con otras culturas.

La propia cuestión de la comunicación plena es una cuestión que se debe pensar, pues en estos años, más que la demanda por la lengua propia, puedo afirmarles que lo que más escuché de los Korúbo es la necesidad de que yo les enseñe el portugués. Además, la lengua oficial del gobierno brasileño es un instrumento de poder que puede no solo empoderar a un grupo específico sino también abrir nuevas puertas y posibilidades a la persona que la domina.

Por su vez, aprender la lengua de un grupo indígena es también empoderar a los actores sociales externos (investigadores, agentes de FUNAI, etc.) frente al grupo, pues ahora ya no existe más un código secreto. El extranjero pasa a tener acceso a códigos verbales y a entendimientos antes imposibles para él y que le garantizaban al grupo comunicarse frente a ellos sin que nadie los entendiera. No hay que olvidar que la persona que pasa a hablar la lengua es también la persona que pasa a filtrar la comunicación entre dos mundos.

El principio de la comunicación plena me llevó a acompañar a los Korúbo en una serie de cuestiones como:

1. negociaciones políticas con FUNAI,

2. internamientos por enfermedades en hospitales en Tabatinga y en Manaus,

3. cacerías y convivencia diaria,

4. negociaciones de enfrentamientos entre ellos,

5. producción de un documental,

6. construcción de un espacio de intercambio,

7. clases formales de Korúbo y de Portugués, 
8. innumerables conversaciones sobre nuestra sociedad y otras cosas que pensamos que podemos explicarles.

Paradójicamente, justo en el momento en que se empieza a delinear el Programa Korúbo es cuando me alejo. Sigo como asesor y apoyo a acciones puntuales. Ahora los Korúbo cuentan también con una asesora en antropología. Hay nuevos actores dentro de un nuevo contexto que deben llevar a cabo acciones junto a los Korúbo. Pero creo que lo principal sigue siendo las determinaciones del grupo frente a la política que perciben que se desarrolla por y para ellos.

Un momento bastante representativo y, tal vez, el más extremo fue el ingreso de los Korúbo contactados al territorio de los Korúbo aislados, en 2011. Hoy es común que los Korúbo contactados digan que los aislados del Coari, donde estuvieron, son un grupo distinto al de ellos, pero aún así no tenemos como determinar si se trata o no del mismo grupo. Las consecuencias para el grupo contactado son perceptibles y se hacen presente diariamente en la huella que permanece en la cabeza de Wanká. La autodeterminación de los Korúbo contactados estaba frente a la autodeterminación de los Korúbo aislados, ¿cómo decidir de cual grupo debería preservarse la autodeterminación?

Mientras diseñamos planes de acción y elaboramos leyes, los diversos grupos nos presentan nuevos desafíos que nos hacen ver que, de hecho, Ramos tiene razón, el indigenismo es "uma obra que nunca se completa". No nos cabe decir cómo se van a realizar las cosas sino trabajar de la mejor manera posible para garantizar los derechos de estos pueblos. En este sentido, hay que buscar que las cosas se realicen con el menor daño para todas las partes involucradas en este proceso llamado contacto.

\section{Una última nota:}

Aunque no aparezca explícitamente en todo el texto, me gustaría lanzar una última observación relacionada a la importancia del componente educativo. Así como sucede con los procesos de los indígenas, por lo general, me parece fundamental que en los trabajos con indígenas en contacto inicial se asegure el componente educativo. Alguien podría preguntarme: ¿Acaso se trata de simplemente enseñarle a un pueblo cómo funciona nuestra sociedad? No, seguramente no es esta la cuestión. Aún que me vengan varias dudas sobre como conducir el proceso de post-contacto, estoy seguro que el componente educativo debe tratar de dar protagonismo a los pueblos indígenas, valorando sus conocimientos y dándoles espacios para elegir y/o seleccionar las cosas que quieren aprender y el como se las interpretan.

A partir de la experiencia Korúbo, me parece que ellos nos exigen que les enseñemos una serie de cosas. Cosas estas que ya deberían estar listas y preparadas cuando del contacto. Pero ¿quién tiene todo listo de los procedimientos para 
el caso de trabajo con indígenas de reciente contacto? Qué yo sepa, nadie. Esta falta de preparación resulta en una necesidad de enseñar y aprender a un mismo tiempo. Entonces, se trata también de generar capacidades en los actores sociales que trabajen directa o indirectamente con estos pueblos. Destacase, por lo tanto, la importancia de los "procesos educativos" como un componente básico y que debe estar presente con esta doble función (enseñar y aprender) desde el comienzo de los trabajos con pueblos de reciente contacto, es decir, tan pronto como sea posible tras el establecimiento del contacto.

\section{Referencias}

Arisi, B. M. 2007. Matis e Korúbo: Contato e índios isolados - relação entre povos no Vale do Javari, Amazônia. Dissertação de Mestrado. Florianópolis: Universidade Federal de Santa Catarina

Coutinho Jr, Walter. 1998. Relatório de identificação e delimitação da Terra Indígena Vale do Javari.Brasília: FUNAI.

Cunha, M. C. da. 1992. "Introdução a uma história indígena". In: Manuela Carneiro da Cunha (org.). História dos Índios no Brasil. São Paulo: Companhia das Letras/ Secretaria Municipal de Cultura.

Freire, C. A. da R. 2005. Sagas sertanistas: práticas e representações do campo indigenista no século XX. Tese de Doutorado. UFRJ/MN - PPGAS: Rio de Janeiro.

Laraia, R. de B. 1986. Cultura: um conceito antropológico. Zahar Editora: Rio de Janeiro.

OACNUDH, 2012. Directrices de protección para los pueblos indígenas en aislamiento y en contacto inicial de la región amazónica, el gran chaco y la región oriental de paraguay. OACNUDH: Genebra.

Oliveira, S. C. S. de. 2009. Preliminares sobre a fonética e a fonologia da lingua falada pelo primeiro grupo de índios Korúbo recém-contatados. Dissertação de Mestrado. UnB: Brasília.

Ramos, A. R. 1999. "Projetos indigenistas no Brasil independente". In: Série Antropologia, 267. UnB - DAN: Brasília.

. 1998. "Uma crítica da desrazão indigenista". In: Série Antropologia, 243. UnB - DAN: Brasília.

Vaz, A. 2011. Politica de Estado: de La Tutela a la Política de Derechos - ¿Una Cuestion Resuelta. Disponível em:

Recebido em setembro de 2016

Aceito em outubro de 2016 